\title{
Dissecting bitcoin blockchain: Empirical Analysis of Bitcoin network (2009-2020)
}

\author{
Pranav Nerurkar ${ }^{\mathrm{a}, \mathrm{b}}$, Dhiren Patel ${ }^{\mathrm{b}}$, Yann Busnel ${ }^{\mathrm{c}}$, Romaric Ludinard ${ }^{\mathrm{c}}$, Saru \\ Kumari $^{\mathrm{d}, *}$, Muhammad Khurram Khan ${ }^{\mathrm{e}}$ \\ ${ }^{a}$ Dept. of Data Science, MPSTME, NMIMS University, Mumbai, India \\ ${ }^{b}$ Dept. of CEEIT, VJTI-Mumbai, India \\ ${ }^{c} S R C D$ department, IMT Atlantique, Rennes, France \\ ${ }^{d}$ Dept. of Mathematics, Ch. Charan Singh University, Meerut, India \\ ${ }^{e}$ Center of Excellence in Information Assurance (CoEIA), King Saud University, Saudi \\ Arabia
}

\begin{abstract}
Bitcoin system (or Bitcoin) is a peer-to-peer and decentralized payment system that uses cryptocurrency named bitcoins (BTCs) and was released as open-source software in 2009. Unlike fiat currencies, there is no centralized authority or any statutory recognition, backing, or regulation for Bitcoin. All transactions are confirmed for validity by a network of volunteer nodes (miners) and after collective agreement is subsequently recorded into a distributed ledger "Blockchain". Bitcoin platform has attracted both social and anti-social elements. On the one hand, it is social as it ensures the exchange of value, maintaining trust in a cooperative, community-driven manner without the need for a trusted third party. At the same time, it is anti-social as it creates hurdles for law enforcement to trace suspicious transactions due to anonymity and privacy. To understand how the social and anti-social tendencies in the user base of Bitcoin affect its evolution, there is a need to analyze the Bitcoin system as a network. The current paper aims to explore the local topology and geometry of the Bitcoin network during its first decade of existence. Bitcoin transaction data from 03 Jan 2009 12:45:05 GMT to 08 May 2020 13:21:33 GMT was processed for this purpose to build a Bitcoin user graph. The characteristics, local and global network properties of the user's graph were analyzed at ten intervals between 2009-2020
\end{abstract}

\footnotetext{
*saryusiirohi@gmail.com
} 
with a gap of one year. Small diameter, skewed distribution of transactions, power-law distributed in and out degrees, disconnected graph, and presence of large connected components were the observations from network analysis. Thus, it could be inferred that despite anti-social tendencies, Bitcoin network shared similarities with other complex networks. Network analysis also uncovered twenty types of legal and anti-social entities operating on Bitcoin and provided a path for uncovering these anti-social entities.

Keywords: Bitcoin, Network Science, Graph Algorithms, Exploratory Data Analysis

1. Introduction

Originally proposed in 2008 by an unknown individual (or a group of individuals) who used a pseudonym "Santoshi Nakamoto", Bitcoin cryptocurrency has since then emerged as the most successful cryptocurrency amongst its peers, reaching an adoption level unrealized by older digital currencies $[1,2,3]$. As on $19^{\text {th }}$ March 2020, Bitcoin has a market cap of USD $\$ 98,584,789,143$ with 18,277,112 bitcoins (BTC's) in circulation each with a value of USD $\$ 5,393.89$. Bitcoin differs from its traditional online banking peers by relying on a decentralized consensus scheme for verifying the correctness and authentic nature of currency transfers between users $[4,5,6]$. The decentralized consensus scheme is made possible by an organized collective of nodes in the Bitcoin system known as "miners". The miners confirm each transaction for authenticity. This increases security in the Bitcoin system and ensures the core philosophy of Bitcoin "Maintain trust in an untrusted environment" without the need for a trusted third party as a reward miners collect transaction fees for the transactions that they confirm.

Illustrating the transaction fundamentals of bitcoin transfers, consider that user $i$ wants to transfer $n$ bitcoins to user $j$. Then $i$ will need a bitcoin wallet, which holds all his private keys and the wallet address of $j$ (Figure 1). Also, the transaction is valid only if user $i$ signs it using his cryptographic key.

Valid transactions are then broadcast over the Bitcoin network, and all miners are informed. Technically, the transaction is not broadcast to all nodes in the Bitcoin network, as a single node can be connected to a maximum 125 (incoming connections $=8$, outgoing connections $=117$ ) other nodes. However, by recursive broadcasts "gossip protocol," a transaction eventually reaches all 


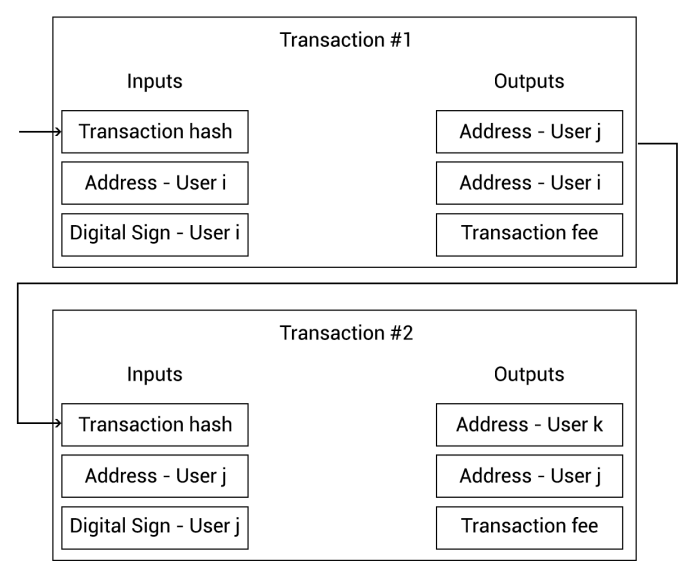

Figure 1: Transfer of bitcoins from user $i$ to $j$ and $j$ to $k$

nodes $[1,7]$. Miners keep all received transactions in their memory pool and combine these transactions to form a "candidate block." Each miner then competes with other miners to add its candidate block to the blockchain. The miner who succeeds gets a reward in BTC's and broadcasts its newly mined block to other miners. Other miners will independently verify the newly mined block before adding it to their blockchain.

Since Bitcoin's inception in 2009, the initial two years saw slow adoption with hardly 1000 unique addresses and less than 10000 transactions per day $[1,8]$. However, as bitcoin became financially significant, there was an exponential growth in transactions from 2012-2016, which also saw the entry of serious users, investors, speculators, and independent mining industries. Before the popularity of bitcoin, the users were mostly crypto-enthusiasts. The change in the profile of Bitcoin's user base was also evident from the increase in the transaction values, fluctuations in BTC price, and volumes of BTC's. This phase also saw the emergence of Ponzi schemes, money laundering, frauds [9], embezzlements, extortion [10] and tax evasion [11] practices that used the blanket of secrecy afforded by Bitcoin to mislead the audit trail. There emerged a diversity even amongst the miners in terms of geography and size. When Bitcoin was launched, it was feasible for any participant to become a miner, but as the user base increased, mining became competitive and required specialized hardware. Miners prefer large warehouses with access to cheap electricity [12]. With time, solo miners decreased and gave way to mining pools. 
As the scale and complexity of the Bitcoin network increased, research interest too emerged to allow for its better understanding $[4,11,12,13,14]$. However, analysis of network properties of Bitcoin graph is an interesting domain, albeit one that has received comparatively less attention. A reason for this could be the complexity of identifying users in the Bitcoin network. In the Bitcoin network, identifying users by wallet addresses (aka accounts, bitcoin addresses, public keys, or other unique identifiers used interchangeably to refer to users' in Bitcoin system) is complicated as these can be generated and discarded multiple times [12]. There is also no upper limit to the identities a single person can create or any limits on the number of transactions or beneficiaries. These factors significantly enhance the hurdles in analyzing the Bitcoin network. To overcome the hurdle caused by multiple identities of a single user, heuristic clustering is applied to the Bitcoin network. With heuristic clustering, multiple identities of a single user are grouped into a single identity. This strategy is used in several Bitcoin network studies $[15,16,17,18]$ and has the advantage of reducing the number of entities of the Bitcoin network.

\subsection{Motivation}

Based on an oft-quoted maxim in network science, "We will never understand complex systems unless we develop a deep understanding of the networks (graphs) behind them" [19], the current paper proposes to shed light on the network properties of Bitcoin. Bitcoin is a diverse ecosystem inhabited by users (wallets) that could be ordinary people interested solely in the exchange of assets or mining nodes competing to ensure that the transactions in their memory pool get added to the blockchain. Though the interactions behind entities in other large systems such as the internet, wireless sensor networks [20, 21, 22], social networking websites, citation systems, file sharing systems are well studied, However Bitcoin system failed to receive similar attention. Network analysis would also help machine learning based applications of Bitcoin such as illegal transaction detection and forensics improve feature engineering.

\subsection{Contributions}

- Conducted a comprehensive study of the large-scale Bitcoin system and interactions occurring in it from 2009 to 2020 by constructing a network from the blockchain files. 
- Studied the Bitcoin network at scale based on local and global graph properties (see Section 3.2).

- Network analysis to uncover types of legal and illegal entities operating on Bitcoin and provide a path for uncovering these entities to aid digital forensic tools.

- Proposed techniques for detection of illegal entities operating in bitcoin network

- Used structural information of Bitcoin network to characterize interactions and evaluate it at scale

- Open sourced the Bitcoin network dataset to motivate independent research

- A time series analysis was performed using previous data obtained of the Bitcoin network. The data for training the machine learning models was from years 2009-2020 and the predictions were made for the year 2021.

So far only I Alqassem et al. [12] and X Lee et al. [13] have provided a detailed graph-theoretic assessment of Blockchain cryptocurrencies. However, $\mathrm{X}$ Lee et al. focused on Ethereum blockchain, and I Alqassem et al. focused on the time period of 2009-2014 to analyze Bitcoin systems. Although these papers provide a technical foundation for the current work, there is no overlap. Ethereum is not just a crypto-currency but also a platform that enables distributed applications. Analysis cannot be compared between Ethereum and Bitcoin. Bitcoin has higher volumes, users and market cap so affects more users and should therefore receive more attention. I Alqassem et al. [12] worked on Bitcoin 2009-2014 so the current papers extended their work to 2020. Additionally, observations and conclusions on future outlook of Bitcoin were made using time series analysis. Time series models are datadriven. So observations and conclusions are obtained after experimentation. The data is allowed to speak for itself and used for predicting growth outlook for year 2021.

The rest of the paper is organized as follows: Section 2 gives the related work done on Bitcoin and other cryptocurrencies. The procedure to convert raw data into a processed form is outlined in Section 3, followed with a description of network analysis tools in Section 3.2 and discussion of results 
in Section 4. The paper concludes in Section 5, mentioning future works for subsequent research.

\section{Related work}

The related work reviewed can be divided into two categories: First, the work that examined the Bitcoin system itself. Second, work that examined other blockchain-based systems.

\subsection{Bitcoin studies}

The journey of Bitcoin, which builds upon nearly two decades of ideas proposed in mailing lists, forum posts, blogs [23], wikis, and source code found in cryptographic circles, is described by $\mathrm{F}$ Tschorsch et al. [14]. However, the authors focused more on framing a tutorial on Bitcoin that includes an outline of selective existing literature. I Alqassem et al. have provided a longitudinal network-based analysis of Bitcoin systems from 2009-2014. The authors have commented upon the changing nature of bitcoin users over time and also drew attention to various structural properties of the Bitcoin system viz. longest connected component, network diameter, densification power law, degree assortativity, time-evolving community structure and inequality in the network [12]. The authors agreed that analyzing the Bitcoin system presents challenges due to the anonymity seeking behaviors of the user base. Though the results highlighted key differences between the Bitcoin network and networks of other systems, the continuous developments and fluctuations in the complex cyber-physical Bitcoin systems necessitate another up-to-date review. T Chang et al. analyzed the various heuristics that are proposed in the literature to identify all public keys that belong to the same user. The heuristics create an approximation of the original Bitcoin network by merging multiple user identifiers to a single identifier and reducing number of entities in the network. Previous studies on network analysis of cryptocurrencies $[12,13,11]$ to have used heuristics and hence, it is a tried and tested method for improving network analysis. S Park et al. scanned the live Bitcoin network for 37 consecutive days in 2018 to track the behavior of the miners. The authors commented upon Bitcoin network statistics such as the number of users, the geographic distribution of users, Bitcoin wallet protocols, and messages propagating in the network [1]. 


\subsection{Studies on other blockchain-based systems}

Y Li et al. used the Ethereum transaction graph (interactions between smart contracts and users) to explore the relationship between the graph structure and crypto-currency price fluctuations [24]. H Sun et al. attempt clustering analysis on Ethereum data to segment malicious users from the rest [25]. S Ferratti et al. has used global network statistical measures such as the order of the network, degree distribution, distance, clustering coefficient, and the tendency of exhibiting a "small world" effect [26]. Based on the observations from these measures, the authors have speculated about the online behavior of Ethereum users, the geographic distribution of miner nodes, and the characteristics of transactions. While S Ferratti et al. argued for the advantages of studying the blockchain structure through a complex network perspective, their focus remained on the Ethereum blockchain structure only. X Lee et al. studied the Ethereum blockchain at scale and applied network analysis measures to characterize interactions between users in Ethereum [13]. The authors studied the network characteristics (vertex count, edge count, self-loop count, and edge density), local network properties (degree distribution, correlation of out and indegree, node centrality measures) and global network properties (reciprocity, assortativity, connected component distribution, diameter, path length, adhesion, cohesion). Just like [26], the authors focused on Ethereum blockchain only but have emphasized that a similar line of network analysis could be extended to another web of blockchain networks. The work in the current paper relies on tools and methods given by S Ferratti et al. [26] and X Lee et al. [13] but targets a longitudinal analysis of Bitcoin network. Table 1 gives the methods and results of network-based studies on blockchain and other real-world systems. 
Table 1: Results of published network studies

\begin{tabular}{|c|c|c|}
\hline $\begin{array}{l}\text { System under } \\
\text { review }\end{array}$ & $\begin{array}{l}\text { Network } \\
\text { theory used }\end{array}$ & Observation \\
\hline Twitter $[27]$ & Gini index & $\begin{array}{l}\text { Dominant nodes are } \\
\text { present }\end{array}$ \\
\hline Facebook [28] & $\begin{array}{l}\text { Assortativity } \\
\text { coefficient }\end{array}$ & Negative assortativity \\
\hline $\begin{array}{l}\text { Social networking } \\
\text { websites }\end{array}$ & $\begin{array}{l}\text { Diameter and } \\
\text { Average path } \\
\text { length }\end{array}$ & Small \\
\hline $\begin{array}{l}\text { Social networking } \\
\text { websites }\end{array}$ & $\begin{array}{l}\text { Clustering } \\
\text { coefficient }\end{array}$ & High \\
\hline $\begin{array}{l}\text { Social networking } \\
\text { websites }\end{array}$ & $\begin{array}{l}\text { Average degree, } \\
\text { Edge density }\end{array}$ & High \\
\hline World wide web $[30,31]$ & Degree distribution & $\begin{array}{l}\text { In and out degree distribution } \\
\text { follow power law }\end{array}$ \\
\hline Protein-protein interaction [31] & Degree distribution & Power law \\
\hline World wide web [32] & Small world effect & $\begin{array}{l}19 \text { hops between any two } \\
\text { webpages }\end{array}$ \\
\hline Facebook $[32,33]$ & $\begin{array}{l}\text { Strongly connected } \\
\text { component (SCC) }\end{array}$ & $\begin{array}{l}99.8 \%-100 \% \text { nodes and } \\
\text { edges are covered. }\end{array}$ \\
\hline Citation networks $[32,33]$ & Graph structure & Acyclic \\
\hline Citation network [30] & Degree distribution & $\begin{array}{l}\text { In and out degree distribution } \\
\text { follow power law }\end{array}$ \\
\hline Film actors [30] & Degree distribution & Power law \\
\hline Company directors [30] & Degree distribution & No power law \\
\hline Co-authorship network [34] & Degree distribution & No power law \\
\hline Ethereum network [13] & $\begin{array}{l}\text { Vertices, arcs, } \\
\text { self-loops, edge density, degree } \\
\text { distributions, centrality } \\
\text { measures, } \\
\text { reciprocity, assortativity, } \\
\text { SCC }\end{array}$ & $\begin{array}{l}\text { In and out degree distribution } \\
\text { follow power law. } \\
\text { Density is low, reciprocity is } \\
\text { positive, assortativity } \\
\text { is negative. SCC has } 98-99 \% \\
\text { nodes and edges. }\end{array}$ \\
\hline D Ding et al. [35] & $\begin{array}{l}\text { Study topological connectivity } \\
\text { and message routability } \\
\text { of } \mathrm{P} 2 \mathrm{P} \text { overlays }\end{array}$ & Degree and Connectivity Analysis \\
\hline D Ding et al. [36] & $\begin{array}{l}\text { Study topological connectivity } \\
\text { and message routability } \\
\text { of P2P overlays }\end{array}$ & Degree and Connectivity Analysis \\
\hline
\end{tabular}

It can be observed from Table 1 that using a unified set of tools and principles, networks of different fields can be studied. This is because, despite variations, networks grow following certain basic principles [37]. 


\section{Bitcoin blockchain to Graph}

Bitcoin blockchain dataset in raw form was obtained from full node at VJTI Blockchain lab ${ }^{1}$. The dataset was of size 268GB and consisted of blockchain in the form of blk.data files. All blocks and transactions from 03 Jan 2009 12:45:05 GMT to 08 May 2020 13:21:33 GMT were present in the dataset. This raw data was then converted to CSV files using the blockchain parser built by the VJTI Blockchain lab ${ }^{2}$. The processed dataset, which is in the form of ".csv" files were made available for download ${ }^{3}$. Table 2 shows the four ".csv" files of the processed dataset.

Table 2: Description of processed dataset

\begin{tabular}{|l|l|l|l|}
\hline Relation & Attributes & \\
\hline Output & tx_hash:START_ID & wallet_address:END_ID & amount \\
\hline Address & wallet_address:ID & & \\
\hline Inputs & wallet_address:START_ID & tx_hash:END_ID & amount \\
\hline Transactions & tx_hash:ID & timestamp & \\
\hline
\end{tabular}

From the Transactions dataset, it is possible to obtain the count of transactions occurring in that year. Each transaction $(\mathrm{tx})$ is identified in blockchain by a unique hash (tx_hash: ID) and has a timestamp, which is the UNIX time of the transaction. For the year 2009, transactions start from 03 Jan 2009 12:45:05 GMT, and for the year 2020, transaction up to 08 May 2020 13:21:33 GMT is considered. Bitcoin entities were identified using an $\mathrm{API}^{4}$ [38]. Table 3 and 4 describes the dataset.

Table 3: Distribution of transactions in Bitcoin blockchain network (2009-2015)

\begin{tabular}{|l|l|l|l|l|l|l|l|}
\hline & $\mathbf{2 0 0 9}$ & $\mathbf{2 0 1 0}$ & $\mathbf{2 0 1 1}$ & $\mathbf{2 0 1 2}$ & $\mathbf{2 0 1 3}$ & $\mathbf{2 0 1 4}$ & $\mathbf{2 0 1 5}$ \\
\hline Transactions & 32741 & 185410 & 1902443 & 8459093 & 19645798 & 25265702 & 45689861 \\
\hline Inputs & 2810 & 108965 & 1902443 & 5716084 & 15407017 & 33300547 & 54564769 \\
\hline Outputs & 32643 & 143863 & 2595309 & 5981241 & 16278420 & 34586691 & 57150816 \\
\hline Max BTC's in a tx & 22500 & 96999 & 550000 & 158336.30 & 194993.50 & 217517.63 & 172841.81 \\
\hline Max inputs in a tx & 320 & 901 & 529 & 673 & 1757 & 674 & 1519 \\
\hline Max outputs in a tx & 2 & 98 & 2002 & 2792 & 3075 & 5352 & 13107 \\
\hline Input sending highest amount & COINBASE & COINBASE & CoinJoin Mess & DeepBit.net & DeepBit.net & Unknown & Unknown \\
\hline Output receiving highest amount & Unknown & Unknown & CoinJoin Mess & DeepBit.net & DeepBit.net & Unknown & Unknown \\
\hline Total BTCs sent & 1978736 & 22667790 & 297984085 & 925215501 & 429732306 & 264107039 & 548006072 \\
\hline
\end{tabular}

\footnotetext{
${ }^{1}$ https://www.vjti-bct.in/

${ }^{2}$ https://github.com/pranavn91/blockchain

${ }^{3}$ https://drive.google.com/open?id=1pEpBAUXKgQX0BP8ircQgd9yXiucLY14h

${ }^{4}$ https://www.walletexplorer.com
} 
Table 4: Distribution of transactions in Bitcoin blockchain network (2016-2020)

\begin{tabular}{|l|l|l|l|l|l|}
\hline & $\mathbf{2 0 1 6}$ & $\mathbf{2 0 1 7}$ & $\mathbf{2 0 1 8}$ & $\mathbf{2 0 1 9}$ & $\mathbf{2 0 2 0}$ \\
\hline Transactions & 82634637 & 104081930 & 81393458 & 119729415 & 39978670 \\
\hline Inputs & 90773554 & 128642149 & 77568478 & 128768057 & 52805351 \\
\hline Outputs & 95783964 & 144361281 & 104780607 & 133558733 & 54179450 \\
\hline Max BTCs in a tx & 99489.99 & 87082.81 & 109735.6 & 157457.612 & 182501 \\
\hline Max inputs in a tx & 677 & 1089 & 1061 & 1347 & 1442 \\
\hline Max outputs in a tx & 11515 & 6626 & 5027 & 7266 & 6990 \\
\hline Input sending highest amount & Unknown & Unknown & Unknown & Unknown & Unknown \\
\hline Output receiving highest amount & Unknown & Unknown & Unknown & Unknown & Unknown \\
\hline Total BTCs sent & 1068404725 & 896026050.66 & 290858051.91 & 515972850.159 & 128637285.824 \\
\hline
\end{tabular}

By parsing through the Bitcoin blockchain dataset, a transaction graph (representing the exchange of bitcoins between wallet addresses) was built. Each transaction has multiple inputs and outputs, as shown in Figure 2. This transaction graph is refined further by heuristic clustering to obtain the user's graph (see Figure 3). The heuristic used for clustering is called the regular inputs heuristic, i.e., all input addresses in a transaction belong to the same user $[5,15]$. The user's graph (payments made between users) leads to meaningful analysis compared to the transaction graph $[15,16,17,18]$. Additionally, the results from the user's graph of Bitcoin can be compared with social network analysis of other real-world systems viz. web, social networking websites, citation graphs. A similar comparison is not possible if the transaction graph of Bitcoin is considered.

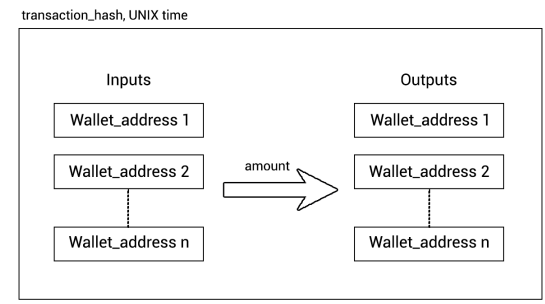

Figure 2: Multi-input multi-output transactions 


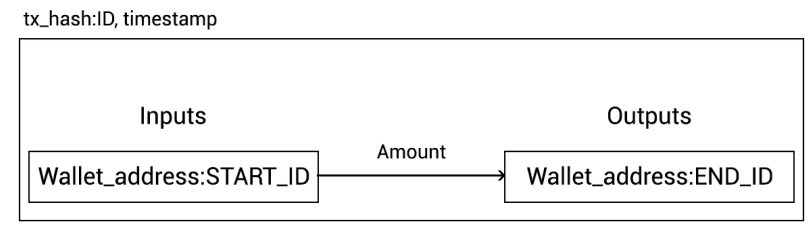

Figure 3: Illustration of attributes of processed dataset

\subsection{Experimental setup}

The preprocessing code is in Python 3.6, and the code for network analysis is in $\mathrm{R}$. The network analysis functions are from the igraph package of $\mathrm{R}$ [39]. The experiments are performed on a single core 1 TB Intel(R) Xeon(R) Silver 4114 CPU@2.20GHz.

\subsection{Network measurements of Bitcoin network}

For this study, Bitcoin user graph is represented as a network $G=(V, E)$, where $V$ refers to the addresses of users' wallets, while $E$ represents a bitcoin exchanges between these wallets. The timestamp of transaction, tx_hash, and amount are attributes of $E$. As multiple transactions can occur between wallet_addresses, $G$ is a directed multi-graph. Using tools described in Section 3.3, an analysis of the Bitcoin network $G$ is performed for the period 2009-2020.

\subsection{Description of tools for Network analysis}

1. Vertex count (order of graph) $|V|$ and edge count (size of graph) $|E|$

2. Graph density $\left(G_{D}\right)$ : Number of edges present graph $G$ amongst all possible edges in $G$. $G_{D}$ for undirected and directed graphs is given by below equations 1 and 2 respectively.

$$
\frac{2|E|}{|V|(|V|-1)}
$$

$$
\frac{|E|}{|V|(|V|-1)}
$$

3. Average degree $d$

$$
d=\frac{1}{|V|} \sum_{u \in V} d(u)=\frac{2 m}{n}
$$


4. Degree distribution of graph $P(k)=\frac{n_{k}}{n}$ is fraction of nodes in the network with degree $k$ i.e. $n_{k}$ where $n$ is the Graph order.

5. Probability distribution

(a) Power law: $y=k^{-\alpha}$ ( $k=$ constant, $\alpha=$ exponent)

(b) Exponential: $y=e^{-\lambda k}(\lambda=$ mean time between events)

(c) Lognormal: $y=\frac{1}{k} e^{-\frac{(\log k-\mu)^{2}}{2 \sigma^{2}}}(\mu=$ scale parameter, $\sigma=$ shape parameter)

(d) Poisson: $\frac{e^{-\mu} \mu^{x}}{x !}$

6. Adhesion or edge connectivity $E$ for connected graph $G$ is the minimum number of edges $\lambda(G)$ whose deletion from a graph $G$ disconnects $G$.

7. cohesion - a minimum number of vertices needed to remove to make the graph not strongly connected

8. Diameter is the length $\max _{(u, v)} d(u, v)$ of the "longest shortest path" (i.e., the longest graph geodesic) between any two graph vertices $(u, v)$ of a graph, where $d(u, v)$ is a graph distance.

9. Average path length $L=\sum_{1}^{E}(G) \frac{d(u, v)}{E(G)}$

10. reciprocity $\rho$ as given in Eq. 4 is the measure of the likelihood of vertices in a directed network to be mutually linked.

$$
\rho=\frac{\sum_{i \neq j\left(a_{i j}-\bar{a}\right)\left(i \neq j\left(a_{j i}-\bar{a}\right)\right.}}{\operatorname{sum}_{i \neq j\left(a_{i j}-\bar{a}\right)^{2}}}
$$

11. Assortativity: level of homophily of the graph.

$$
r=\frac{\sum_{j k} j k\left(e_{j k}-q_{j} q_{k}\right)}{\sigma_{q}^{2}}
$$

where,

- $q_{k}$ number of edges leaving the node, other than the one that connects the pair $j, k$

- $\sigma_{q}$ standard deviation of q in Eq. 5 
- $e_{j k}$ refers to the joint probability distribution of the remaining degrees of the two vertices

12. Number of connected components of a graph $G$ is $c(G)$. A connected component is a set of vertices all of which are connected, and unconnected to the other nodes in the network. The weakly connected components are found by performing breadth-first search. The strongly connected components are implemented by two consecutive depth-first searches.

13. Degree Centrality of a vertex $v_{i}$ is defined as $\operatorname{deg}\left(v_{i}\right) / 2|E|$

14. Betweenness centrality $C_{B}(v)$ of $v \in V$ is the fraction of times $v$ occurs on any shortest path connecting any other pair of vertices $s, t \in V$. Let $\sigma_{s t}$ be the total number of shortest paths connecting vertex $s$ with vertex $t$. Let $\sigma_{s t}(v)$ be the number of these shortest paths containing $v$. The geodesic centrality of $v$ is:

$$
C_{B}(v)=\sum_{s \neq t \neq v} \frac{\sigma_{s t}(v)}{\sigma_{s t}}
$$

15. Size of largest strongly connected component $N_{s}$ - a set of vertices in a directed graph such that any node is reachable from any other node using a path following only directed edges in the forward direction.

$$
\begin{aligned}
N & =\max _{F \subseteq \mathcal{C}}|F| \\
\mathcal{C} & =\left\{C \subseteq V \mid \forall u, v \in C: \exists w_{1}, w_{2}, \ldots \in V: u \sim w_{1} \sim w_{2} \sim \cdots \sim v\right\}
\end{aligned}
$$

16. Relative size of the largest connected component $\left(N_{\text {rel }}\right)$ equals the size of the largest connected component divided by the size of the network

$$
N_{\text {rel }}=\frac{N}{n} .
$$

17. Number of triangles defined in the following way is independent of the orientation of edges when the graph is directed.

$$
t=|\{\{u, v, w\} \mid u \sim v \sim w \sim u\}| / 6
$$


18. Global clustering of a network is the probability that two incident edges are completed by a third edge to form a triangle

$$
c=\frac{|\{u, v, w \in V \mid u \sim v \sim w \sim u\}|}{|\{u, v, w \in V \mid u \sim v \neq w \sim u\}|}
$$

Tools for network measurement can be divided into three groups: measures for characteristics (vertex count, edge count, edge density), measures of local network properties (radius, local clustering coefficient, node degree) and measures for global network properties (degree distribution, adhesion, cohesion, components, centralization, k-cores).

\section{Experimental study}

Bitcoin users graph is studied using the tools given in Section 3.3. The entire Bitcoin network is studied at eleven intervals, as seen in the results. The year in the results corresponds to a Bitcoin users graph built from transaction data considered from 01 Jan 12:00:00 AM GMT to 31 Dec 11:59:59 PM GMT of that year. An exception is the year 2020, which is built using transaction data from 01 Jan 2020 12:00:00 AM GMT to 08 May 2020 13:21:33 GMT.

\subsection{Bitcoin Network characteristics}

Table 5 gives the bitcoin users graph. Two versions of edge density are indicated by $(\mathrm{S})$ for a simple, undirected version of the user's graph and (D) for the directed user's graph. Multiple directed edges between two users are collapsed to a single undirected edge to obtain edge density (S). Vertex count in Table 5 and 6 gives the total senders and receivers in that calendar year. Bitcoin users have increased till 2017, leading to the price of BTC's reaching its peak in Dec 2017. The following years have seen a decline in both users and the value of BTCs. In 2009, out of 32741 transactions, 32522 were COINBASE transactions. The highest number of BTCs transferred in a single transaction was 22500, and 320 were the highest number of inputs present in a transaction. Limited edges were created as transactions between users were less. The edge density is low in both the directed graph (Edge density (D)) and the undirected graph (Edge density (S)) for the period 2009-2020 compared to social networks. The low density is due to the skewed distribution of transactions amongst the users. $99.8 \%$ of the total users in 2009 made almost a single transaction. This declined to $73.24 \%$ by 2020 . 
Table 5: Characteristics of Bitcoin blockchain network (2009-2015)

\begin{tabular}{|l|l|l|l|l|l|l|l|}
\hline & $\mathbf{2 0 0 9}$ & $\mathbf{2 0 1 0}$ & $\mathbf{2 0 1 1}$ & $\mathbf{2 0 1 2}$ & $\mathbf{2 0 1 3}$ & $\mathbf{2 0 1 4}$ & $\mathbf{2 0 1 5}$ \\
\hline Vertex count & 32644 & 143943 & 2599119 & 6001831 & 16337189 & 34693993 & 57381025 \\
\hline Edge count & 32808 & 233872 & 4642054 & 19710026 & 49336100 & 78077032 & 145496703 \\
\hline Edge density (S) & $6.16 \mathrm{e}-05$ & $2.25 \mathrm{e}-05$ & $1.28 \mathrm{e}-07$ & $3.4 \mathrm{e}-07$ & $0.94 \mathrm{e}-07$ & $3.7 \mathrm{e}-08$ & $2.37 \mathrm{e}-08$ \\
\hline Edge density (D) & $3.08 \mathrm{e}-05$ & $1.12 \mathrm{e}-05$ & $6.87 \mathrm{e}-07$ & $5.4 \mathrm{e}-07$ & $1.85 \mathrm{e}-07$ & $6.48 \mathrm{e}-08$ & $4.42 \mathrm{e}-08$ \\
\hline
\end{tabular}

Table 6: Characteristics of Bitcoin blockchain network (2016-2020)

\begin{tabular}{|l|l|l|l|l|l|}
\hline & $\mathbf{2 0 1 6}$ & $\mathbf{2 0 1 7}$ & $\mathbf{2 0 1 8}$ & $\mathbf{2 0 1 9}$ & $\mathbf{2 0 2 0}$ \\
\hline Vertex count & 57107986 & 78724132 & 53049193 & 32288199 & 3160555 \\
\hline Edge count & 29365348 & 625420597 & 330885984 & 230911982 & 24840651 \\
\hline Edge density (S) & $5.2 \mathrm{e}-08$ & $0.49 \mathrm{e}-07$ & $0.68 \mathrm{e}-07$ & $1.12 \mathrm{e}-07$ & $1.18 \mathrm{e}-06$ \\
\hline Edge density (D) & $9 \mathrm{e}-08$ & $1.01 \mathrm{e}-07$ & $1.17 \mathrm{e}-07$ & $2.21 \mathrm{e}-07$ & $2.49 \mathrm{e}-06$ \\
\hline
\end{tabular}

Till the year 2010, Bitcoin was used by crypto-enthusiasts and year 2011 saw the entry of the first mixing service and mining pools. Both these services involve transactions with one or limited inputs and several outputs. Consequentially, the maximum number of outputs in a single transaction increased from 98 in 2010 to 2002 in 2011 and has remained in range of 2000-7000. This leads to observation that "Number of outputs" can be used to discriminate between different types of users in Bitcoin.

\subsection{Vertex degree distribution}

The procedure mentioned by C Gillespie [40] was followed to understand the distribution of in (see Table 7 and 8) and out degrees (Table 9 and 10) of users graph. In 2009, for the distribution of in degrees, the minimum value from which the power-law distribution was fitted i.e., $\left(x_{\min }\right)$ was 4 and for exponential $x_{\min }$ was 1 , log-normal $x_{\min }$ was 1 and poission $x_{\min }$ was 5 . For 2010, $x_{\min }$ was 31 for power law, 183 for exponential, 29 for log-normal and 4351 for poisson. In 2011, $x_{\min }$ was 397 for power law, 279 for exponential, 359 for log-normal and 8079 for poisson. In 2012, $x_{\text {min }}$ was 621 for power law, 72053 for exponential, 608 for log-normal and 5352 for poisson. In 2013, $x_{\min }$ was 987 for power law, 76728 for exponential, 1151 for log-normal and 4751 for poisson. In 2014, $x_{\min }$ was 1615 for power law, 99867 for exponential, 1702 for log-normal and 154 for poisson. In 2015, $x_{\min }$ was 2994 for power law, 99891 for exponential, 1950 for log-normal and 359 for poisson. 
Table 7: Likelihood ratio tests for comparing in degree distribution (2009-2015)

\begin{tabular}{|l|l|l|l|l|l|l|l|l|}
\hline Distributions & Parameters & $\mathbf{2 0 0 9}$ & $\mathbf{2 0 1 0}$ & $\mathbf{2 0 1 1}$ & $\mathbf{2 0 1 2}$ & $\mathbf{2 0 1 3}$ & $\mathbf{2 0 1 4}$ & $\mathbf{2 0 1 5}$ \\
\hline Power law & $\alpha$ & 1.99 & 1.54 & 2.35 & 1.86 & 1.88 & 1.98 & 2.12 \\
\hline Exponential & $\lambda$ & 0.11 & 0.001 & 0.011 & 0.004 & 0.002 & 0.002 & 0.0001 \\
\hline \multirow{2}{*}{ Log-normal } & $\mu$ & 1.79 & 2.59 & -26.61 & -52.63 & -29.818218 & -21.38 & 2.62 \\
\cline { 2 - 8 } & $\alpha$ & 1.01 & 2.65 & 5.06 & 8.42 & 6.50 & 5.55 & 2.61 \\
\hline Poisson & $\mu$ & 13.83 & 4992.6 & 26133.67 & 43568.6 & 43778.7 & 7764.21 & 8610.67 \\
\hline
\end{tabular}

In $2016, x_{\min }$ was 2318 for power law, 99549 for exponential, 1510 for log-normal and 5 for poisson. In 2017, $x_{\min }$ was 3118 for power law, 99671 for exponential, 99671 for log-normal and 6294 for poisson. In 2018, $x_{\min }$ was 1862 for power law, 96500 for exponential, 2179 for log-normal and 11175 for poisson. In 2019, $x_{\text {min }}$ was 2674 for power law, 97258 for exponential, 97258 for log-normal and 1 for poisson. In 2020, $x_{\min }$ was 2588 for power law, 95384 for exponential, 1939 for log-normal and 1 for poisson. From Table 7 it is observed that power-law and log-normal are better fit to data than exponential or poisson. Moreover, $X_{\min }$ values indicate that tail of the distribution follows power law. $\alpha$ value indicates inverse relationship between degree and frequency of such nodes. High degree nodes such as mixing services and pools would form LSCC/LWCC making it easy for identifying them on Bitcoin.

Table 8: Likelihood ratio tests for comparing in degree distribution (2016-2020)

\begin{tabular}{|l|l|l|l|l|l|l|}
\hline Distributions & Parameters & $\mathbf{2 0 1 6}$ & $\mathbf{2 0 1 7}$ & $\mathbf{2 0 1 8}$ & $\mathbf{2 0 1 9}$ & $\mathbf{2 0 2 0}$ \\
\hline Power law & $\alpha$ & 2.1 & 2.11 & 1.92 & 2.4 & 2.2 \\
\hline Exponential & $\lambda$ & 0.001 & 0.001 & 0.001 & 0.001 & 0.003 \\
\hline \multirow{2}{*}{ Log-normal } & $\mu$ & 5.15 & -194.65 & -17.11 & -398.36 & -7.01 \\
\cline { 2 - 6 } & $\alpha$ & 2.06 & 12.1 & 5.29 & 15.85 & 3.78 \\
\hline Poisson & $\mu$ & 7918 & 29039.39 & 63050.8 & 5095.25 & 4054.3 \\
\hline
\end{tabular}

In 2009, for the distribution of out degrees, the minimum value from which the power-law distribution was fitted i.e., $\left(x_{\min }\right)$ was 4 and for exponential $x_{\min }$ was $3, \log$-normal $x_{\min }$ was 1 and poission $x_{\min }$ was 12 . For $2010, x_{\min }$ was 14 for power law, 5136 for exponential, 15 for log-normal and 42 for poisson. In 2011, $x_{\text {min }}$ was 520 for power law, 42350 for exponential, 145 for log-normal and 252 for poisson. In 2012, $x_{\min }$ was 667 for power law, 93316 for exponential, 562 for log-normal and 2210 for poisson. In 2013, $x_{\min }$ was 1073 for power law, 94828 for exponential, 94828 for log-normal and 2244 for poisson. In $2014, x_{\min }$ was 1540 for power law, 98344 for exponential, 1544 for log-normal and 2334 for poisson. In 2015, $x_{\min }$ was 2251 for power law, 98992 for exponential, 2214 for log-normal and 300 for poisson. 
Table 9: Likelihood ratio tests for comparing out degree distribution (2009-2015)

\begin{tabular}{|l|l|l|l|l|l|l|l|l|}
\hline Distributions & Parameters & $\mathbf{2 0 0 9}$ & $\mathbf{2 0 1 0}$ & $\mathbf{2 0 1 1}$ & $\mathbf{2 0 1 2}$ & $\mathbf{2 0 1 3}$ & $\mathbf{2 0 1 4}$ & $\mathbf{2 0 1 5}$ \\
\hline Power law & $\alpha$ & 1.33 & 1.42 & 1.73 & 1.74 & 1.85 & 1.86 & 1.87 \\
\hline Exponential & $\lambda$ & 0.25 & 0.06 & 0.013 & 0.005 & 0.002 & 0.002 & 0.001 \\
\hline \multirow{2}{*}{ Log-normal } & $\mu$ & -7.27 & -4.52 & -52.81 & -7.835970 & -137.41132 & -18.89 & 1.25 \\
\cline { 2 - 8 } & $\alpha$ & 6.10 & 5.14 & 8.31 & 4.77 & 10.3 & 5.73 & 3.14 \\
\hline Poisson & $\mu$ & 10851.33 & 3754.7 & 4516.74 & 27558.8 & 24466.7 & 25145.02 & 14322.95 \\
\hline
\end{tabular}

In 2016, $x_{\min }$ was 2224 for power law, 99977 for exponential, 1722 for lognormal and 2314 for poisson. In 2017, $x_{\min }$ was 5338 for power law, 96639 for exponential, 2820 for log-normal and 1 for poisson. In 2018, $x_{\min }$ was 4308 for power law, 97340 for exponential, 6600 for log-normal and 10649 for poisson. In 2019, $x_{\min }$ was 9124 for power law, 98154 for exponential, 98154 for log-normal and 1 for poisson. In 2020, $x_{\min }$ was 842 for power law, 84442 for exponential, 456 for log-normal and 69 for poisson.

Table 10: Likelihood ratio tests for comparing out degree distribution (2016-2020)

\begin{tabular}{|l|l|l|l|l|l|l|}
\hline Distributions & Parameters & $\mathbf{2 0 1 6}$ & $\mathbf{2 0 1 7}$ & $\mathbf{2 0 1 8}$ & $\mathbf{2 0 1 9}$ & $\mathbf{2 0 2 0}$ \\
\hline Power law & $\alpha$ & 1.77 & 2.58 & 2.34 & 2.7 & 2.07 \\
\hline Exponential & $\lambda$ & 0.001 & 0.001 & 0.0006 & 0.0007 & 0.0051 \\
\hline \multirow{2}{*}{ Log-normal } & $\mu$ & 7.3 & 7.76 & 4.8 & -338.17 & 5.56 \\
\cline { 2 - 6 } & $\alpha$ & 1.8 & 1.13 & 2.02 & 11.65 & 1.67 \\
\hline Poisson & $\mu$ & 15859.95 & 5967.4 & 28175.95 & 5362.98 & 2580.6 \\
\hline
\end{tabular}

Figure 4 and 5 show the fitting of four heavy-tailed distributions to indegree and out-degree distribution of users graph respectively. Four distributions considered are discrete power law (red), exponential (dark blue), lognormal (green), and Poisson (light blue). Distribution is fit as per protocol specified by C Gillespie [40]. 


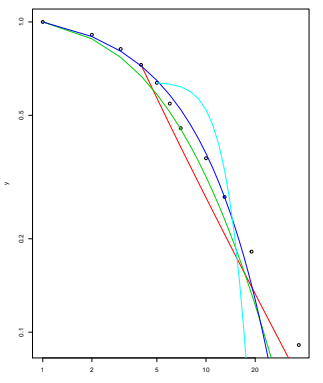

(a) 2009

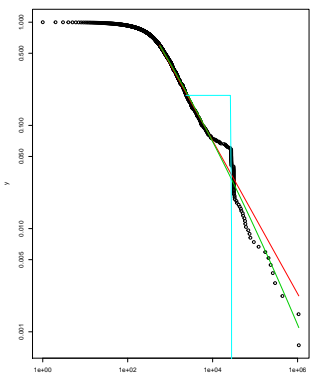

(d) 2012

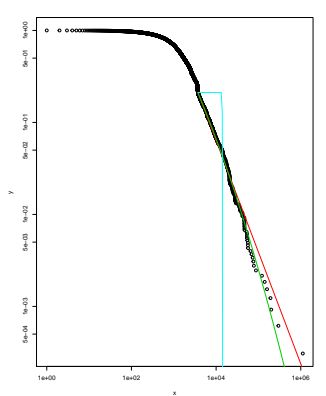

(g) 2015

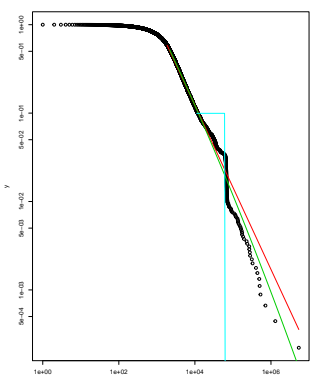

(j) 2018

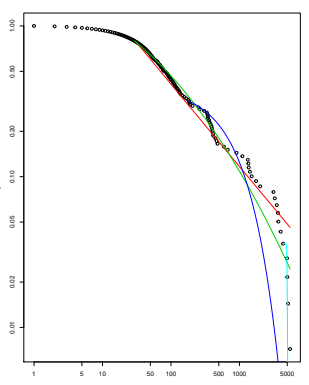

(b) 2010

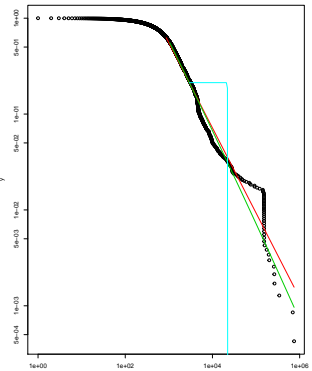

(e) 2013

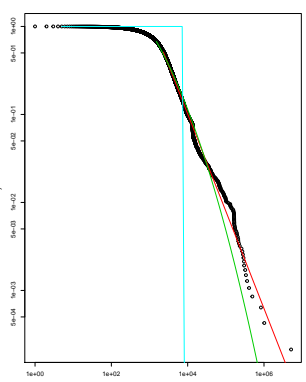

(h) 2016

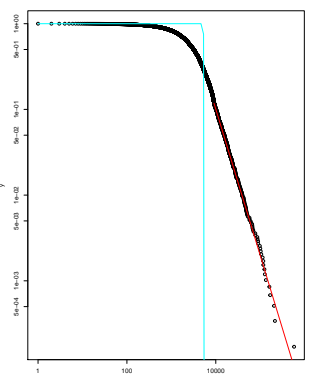

(k) 2019

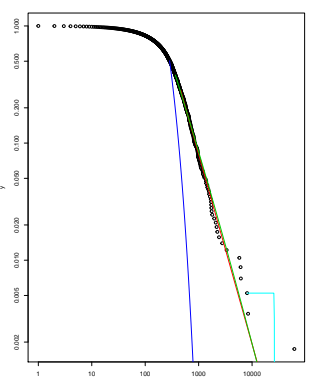

(c) 2011

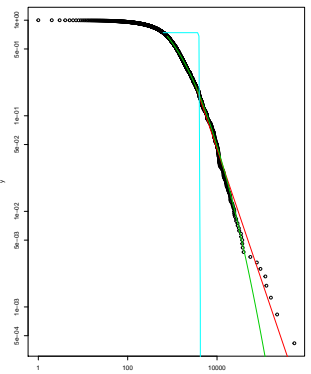

(f) 2014

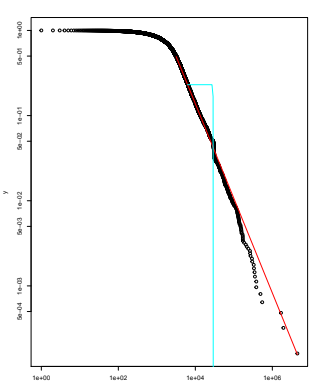

(i) 2017

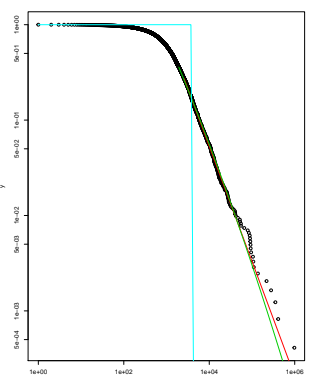

(l) 2020

Figure 4: In-degree distribution of Bitcoin users graph (2009-2020) 


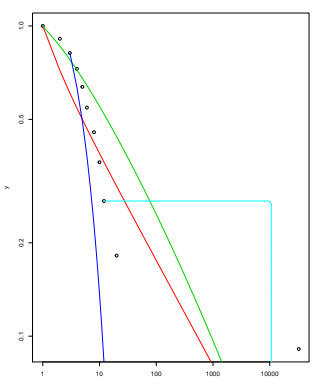

(a) 2009

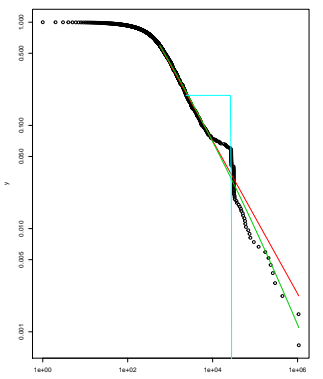

(d) 2012

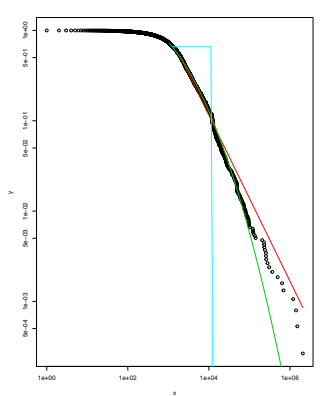

(g) 2015

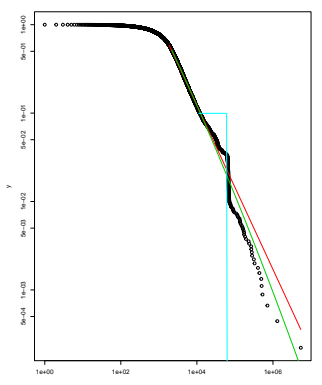

(j) 2018

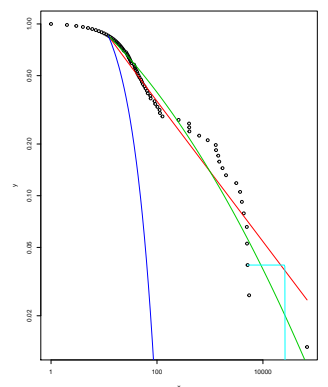

(b) 2010

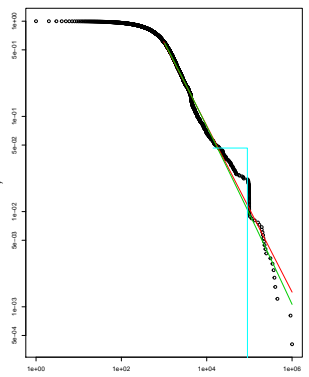

(e) 2013

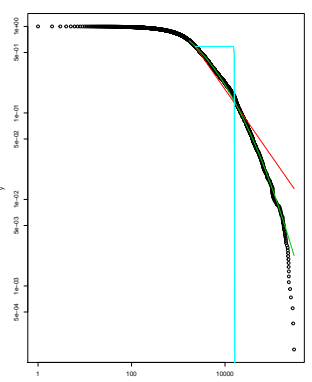

(h) 2016

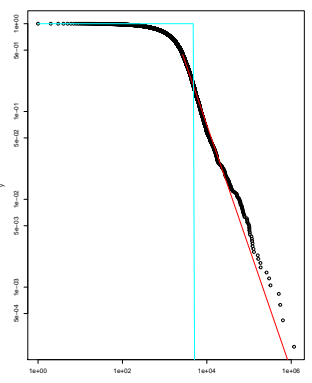

(k) 2019

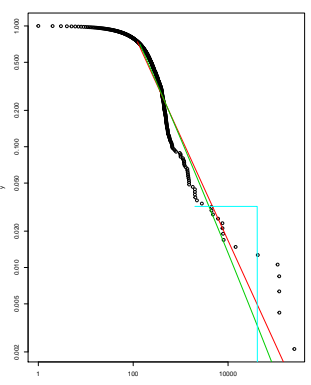

(c) 2011

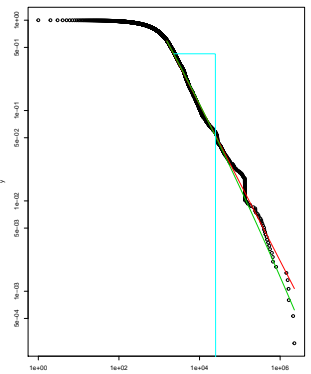

(f) 2014

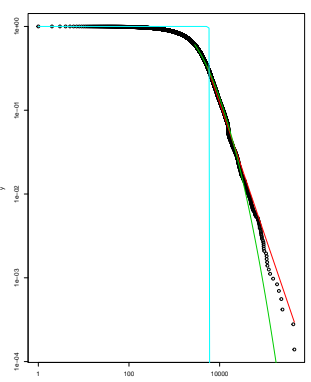

(i) 2017

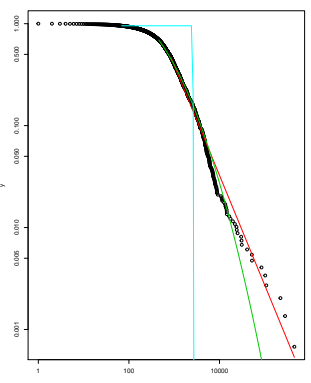

(l) 2020

Figure 5: Out-degree distribution of Bitcoin users graph (2009-2020) 
As claimed for most complex networks, even bitcoin users graph followed the "scale-free" property as power-law exponent ranged from 1.54-2.4 for in-degree distribution and from 1.42-2.7 for out-degree distribution. $x_{\text {min }}$ indicated that the tail of the in and out-degree distributions fit the power law. High degree entities such as mixing services, gambling websites and pools will occupy the tail of the degree distribution. Whereas, ordinary users shall be at the other end of the spectrum. Thus, the location of the entity on the degree distribution curve could reveal its nature.

\subsection{Bitcoin: Global networks properties}

Table 11 and 12 give the global network properties of bitcoin users graph. Measures marked with \# could not be computed on the current configuration of the system.

indicates approximation used for computation as given by M Jackson et al. [41]. In 2009, as transactions were infrequent, adhesion and cohesion were zero indicating a sparsely connected graph where information transfer was slow due to long diameter. As the majority were COINBASE transactions in 2009, the graph had high centralization tendency, low reciprocity, girth, and assortativity. Till 2010, crypto-enthusiasts dominated the transactions, and transactions were less, and diameter increased. In 2011, mixing services and miner pools entered, and the DeepBit.net mining pool had 61897 incoming and 120756 outgoing connections. CoinJoin Mess, a mixing service, had 903 incoming and 1800 outgoing connections in 2011. The presence of mining pools and mixing services decreased the diameter and average path length while leading an increase in reciprocity. In 2012, SantoshiDice.com, a gambling website, saw 810474 incoming and 1055385 outgoing connections. In 2013 too SantoshiDice.com continued to get the highest incoming and outgoing connections. In 2014, SantoshiDice.com had the maximum incoming connections (1592352), whereas CoinJoin Mess had the maximum outgoing (2256302). In 2015, another online gambling site LuckyBit.it had the highest incoming connections at 1655881, and CoinJoinMess had the highest outgoing connections at 2256344 . 
Table 11: Global network properties (2009-2015)

\begin{tabular}{|l|l|l|l|l|l|l|l|}
\hline & $\mathbf{2 0 0 9}$ & $\mathbf{2 0 1 0}$ & $\mathbf{2 0 1 1}$ & $\mathbf{2 0 1 2}$ & $\mathbf{2 0 1 3}$ & $\mathbf{2 0 1 4}$ & $\mathbf{2 0 1 5}$ \\
\hline Adhesion & 0 & 0 & 0 & 0 & 0 & 0 & 0 \\
\hline Cohesion & 0 & 0 & 0 & 0 & 0 & 0 & 0 \\
\hline Diameter & 7 & 5525 & $0.03^{+}$ & $0.06^{+}$ & $0.06^{+}$ & $0.05^{+}$ & $0.05^{+}$ \\
\hline Average path & 1.01 & 748.54 & $0.03^{+}$ & $0.06^{+}$ & $0.06^{+}$ & $0.05^{+}$ & $0.05^{+}$ \\
\hline Radius & 6 & 1 & $\#$ & $\#$ & $\#$ & $\#$ & $\#$ \\
\hline Reciprocity & $6.11 \mathrm{e}-05$ & 0.02 & 0.008 & 0.2 & 0.16 & 0.03 & 0.019 \\
\hline Girth & 3 & 3 & 3 & 3 & 3 & 3 & 3 \\
\hline Assortativity & -0.55 & -0.31 & 0.17 & 0.12 & 0.06 & 0.04 & 0.17 \\
\hline Centralization & 0.99 & 1 & 0.99 & 0.99 & 0.99 & 1 & 1 \\
\hline$C_{d}$ & 0.5 & 0.23 & 0.04 & 0.15 & 0.05 & 0.03 & 0.02 \\
\hline$C_{c}$ & $0.99^{*}$ & $2.1 \mathrm{e}-06$ & $\#$ & $\#$ & $\#$ & $\#$ & $\#$ \\
\hline
\end{tabular}

In 2016, with 300120 outgoing connections, Faucetbox.com (bitcoin reward site) was very active. In 2017 highest connections were recorded by Poloniex.com, a crypto exchange with 4473190 incoming and 445628 outgoing connections. In 2019, Huobi.com-2, a bitcoin exchange platform, had the highest outgoing connections. Due to anonymity, the identity of an entity with the highest incoming and outgoing connections in 2018 was not found.

Table 12: Global network properties (2016-2020)

\begin{tabular}{|l|l|l|l|l|l|}
\hline & $\mathbf{2 0 1 6}$ & $\mathbf{2 0 1 7}$ & $\mathbf{2 0 1 8}$ & $\mathbf{2 0 1 9}$ & $\mathbf{2 0 2 0}$ \\
\hline Adhesion & 0 & 0 & 0 & 0 & 0 \\
\hline Cohesion & 0 & 0 & 0 & 0 & 0 \\
\hline Diameter & $0.09^{+}$ & $0.11^{+}$ & $0.1^{+}$ & $0.11^{+}$ & $0.13^{+}$ \\
\hline Average path & $0.09^{+}$ & $0.11^{+}$ & $0.1^{+}$ & $0.11^{+}$ & $0.13^{+}$ \\
\hline Radius & $\#$ & $\#$ & $\#$ & $\#$ & $\#$ \\
\hline Reciprocity & 0.016 & 0.003 & 0.0016 & 0.0009 & 0 \\
\hline Girth & 3 & 3 & 3 & 3 & 3 \\
\hline Assortativity & -0.026 & -0.005 & -0.022 & 0.28 & 0.09 \\
\hline Centralization & 0.99 & 0.99 & 0.99 & 1 & 0 \\
\hline$C_{d}$ & 0.044 & 0.031 & 0.05 & 0.02 & 0.15 \\
\hline$C_{c}$ & $\#$ & $\#$ & $\#$ & $\#$ & $\#$ \\
\hline
\end{tabular}

404 exchanges, pools, mixers). Due to the high transactions received by such entities the centralization remained close to 1 . Based on these observations, 
transaction based features would be key in discriminating entities. These features would be - Total transactions in which wallet has participated $\left(T_{x}\right)$, Total incoming transactions to the wallet $\left(T_{x}^{i n}\right)$, Total outgoing transactions from the wallet $\left(T_{x}^{\text {out }}\right)$, Average number of incoming transactions received by an address of a wallet $\left(A_{v}\right)$, Total number of addresses sending BTC to the wallet $(T)$ and Ratio of Transaction count and address count $(R)$ gives the average number of times an address of the wallet was reused for a transaction.

\subsection{Community structure}

Usually, triangles, transitivity, and clustering coefficient are higher in social networks than non-social networks [13]. These parameters indicate the tendency of entities in the network to form dense communities. In 2009, the Largest Weakly Connected Component (LWCC) was the entire graph, and Largest Strongly Connected Component (LSCC) was minimal. Triangles and clustering coefficients were also negligible. In 2010, WCC was 25, and SCC was 108482. In 2011, WCC was 1400, and SCC were 2029127. In 2012, WCC was 6165, and SCC were 3149100. In 2013, WCC was 15122, and SCC was 9888167. DeepBit.net formed the largest SCC and largest WCC in 2011. SantoshiDice.com formed the largest SCC and largest WCC in 2012 (see Table 13).

Table 13: Community structure (2009-2012)

\begin{tabular}{|l|l|l|l|l|l|}
\hline & & $\mathbf{2 0 0 9}$ & $\mathbf{2 0 1 0}$ & $\mathbf{2 0 1 1}$ & $\mathbf{2 0 1 2}$ \\
\hline \multirow{5}{*}{ LSCC } & Triangles & 0 & 9580 & 104368 & 3797352 \\
\cline { 2 - 6 } & Nodes & $2(0 \%)$ & $34709(24.1 \%)$ & $567144(21.8 \%)$ & $2846171(47 \%)$ \\
\cline { 2 - 6 } & Edges & $5(0 \%)$ & $75367(32.2 \%)$ & $1345036(28.9 \%)$ & $13908941(70 \%)$ \\
\cline { 2 - 6 } & Articulation pt. & 0 & 72 & 638 & 1389 \\
\cline { 2 - 5 } & $\mathrm{C}$ & $\mathrm{NaN}$ & 0.003 & 0.003 & $9.1 \mathrm{e}-05$ \\
\hline \multirow{5}{*}{ LWCC } & Triangles & 9 & 18708 & 3102649 & 4267711 \\
\cline { 2 - 6 } & Nodes & $32644(100 \%)$ & $143880(100 \%)$ & $2593961(100 \%)$ & $5979901(100 \%)$ \\
\cline { 2 - 6 } & Edges & $32808(100 \%)$ & $233829(100 \%)$ & $4638181(100 \%)$ & $19693726(100 \%)$ \\
\cline { 2 - 5 } & Articulation pt. & 79 & 20774 & 496060 & 1440988 \\
\cline { 2 - 6 } & C & $2.4 \mathrm{e}-05$ & $1.11 \mathrm{e}-05$ & 0.0005 & 0.0001 \\
\hline \multirow{5}{*}{ Full network } & Triangles & 9 & 18709 & 3102700 & 4267910 \\
\cline { 2 - 6 } & Articulation pt. & 79 & 20784 & 497641 & 1447747 \\
\cline { 2 - 5 } & C & $2.4 \mathrm{e}-05$ & $1.11 \mathrm{e}-05$ & 0.0005 & 0.0001 \\
\hline
\end{tabular}

In 2013, 2014 and 2015 too the largest SCC and WCC were formed by SantoshiDice.com (see Table 14). In 2014, there were a total of 40508 WCC and 24516983 SCC in the network. In 2015, WCC was 253244, and SCC were 35766309 in the network. 
Table 14: Community structure (2013-2015)

\begin{tabular}{|l|l|l|l|l|}
\hline & & $\mathbf{2 0 1 3}$ & $\mathbf{2 0 1 4}$ & $\mathbf{2 0 1 5}$ \\
\hline \multirow{5}{*}{ LSCC } & Triangles & 7751768 & 5140336 & 21461343 \\
\cline { 2 - 5 } & Nodes & $6437119(39.4 \%)$ & $10157747(29.6 \%)$ & $17445491(30.2 \%)$ \\
\cline { 2 - 5 } & Edges & $32501745(65.8 \%)$ & $41139689(52.3 \%)$ & $85078065(58.9 \%)$ \\
\cline { 2 - 5 } & Articulation pt. & 9270 & 14777 & 14790 \\
\cline { 2 - 5 } & $\mathrm{C}$ & 0.0002 & 0.0008 & 0.0004 \\
\hline \multirow{5}{*}{ LWCC } & Triangles & 7751768 & 6832830 & 25928531 \\
\cline { 2 - 5 } & Nodes & $16282225(100 \%)$ & $34556782(100 \%)$ & $57084066(100 \%)$ \\
\cline { 2 - 5 } & Edges & $49292728(100 \%)$ & $77961419(100 \%)$ & $145254102(100 \%)$ \\
\cline { 2 - 5 } & Articulation pt. & 4282322 & 7775376 & 13682985 \\
\cline { 2 - 5 } & C & 0.0002 & 0.0001 & 0.0002 \\
\hline \multirow{5}{*}{ Full network } & Triangles & 9102472 & 6834251 & 25931343 \\
\cline { 2 - 5 } & Articulation pt. & 4297982 & 7809891 & 13771043 \\
\cline { 2 - 5 } & C & 0.0002 & 0.0001 & 0.0002 \\
\hline
\end{tabular}

In 2016, unknown wallets had formed the largest WCC and SCC. In 2017, Bittrex.com, a crypto trading exchange, formed the largest SCC. In 2019, the largest SCC was formed by Bitcoin exchange service Huobi.com-2. In 2016, WCC was 871640, and SCC was 46385054 in the network. In 2017, WCC was 1476165 , and SCC were 69375203. In 2018, WCC was 1032588, and SCC were 30074974. In 2019, WCC were 967845 and SCC were 26896674 (see Table 15).

Table 15: Community structure (2016-2020)

\begin{tabular}{|c|c|c|c|c|c|c|}
\hline & & 2016 & 2017 & 2018 & 2019 & 2020 \\
\hline \multirow{5}{*}{ LSCC } & Triangles & 125423937 & 95674389 & 62367145 & 24089648 & 0 \\
\hline & Nodes & $10698736(18.7 \%)$ & $9306342(3 \%)$ & $3242666(6.1 \%)$ & $844423(2.7 \%)$ & 1 \\
\hline & Edges & $120658573(41.1 \%)$ & $169589795(15.07 \%)$ & $62330136(18.8 \%)$ & $18010394(8.2 \%)$ & 0 \\
\hline & Articulation pt. & 1259 & 2206 & 717 & 522 & 0 \\
\hline & $\mathrm{C}$ & 0.0015 & 0.0009 & 0.0004 & 0.004 & 0 \\
\hline \multirow{5}{*}{ LWCC } & Triangles & 213985326 & 210765433 & 214016097 & 88648952 & 0 \\
\hline & Nodes & $53556287(93.7 \%)$ & $74366786(94.4 \%)$ & $47785524(90.7 \%)$ & $26470992(85.5 \%)$ & $123583(0.03 \%)$ \\
\hline & Edges & $287695383(93.7 \%)$ & $618579809(98.9 \%)$ & $325783461(98.4 \%)$ & $212922543(97.8 \%)$ & $403262(0.01 \%)$ \\
\hline & Articulation pt. & 5333181 & 6854728 & 4535938 & 3167225 & 4785 \\
\hline & $\mathrm{C}$ & 0.0005 & 0.0003 & 0.0001 & 0.0004 & 0 \\
\hline \multirow{3}{*}{ Full network } & Triangles & 214055511 & 287646955 & 214094259 & 88721557 & 0 \\
\hline & Articulation pt. & 6212728 & 6987676 & 5488866 & 4060330 & 351463 \\
\hline & $\mathrm{C}$ & 0.0005 & 0.0003 & 0.0001 & 0.0004 & 0 \\
\hline
\end{tabular}

The LSCC increased from 2009-2012 to close to $47 \%$ of all nodes of the graph in 2012 and then has declined to $2-3 \%$ of all nodes by 2019. LWCC has remained in a range of $97-98 \%$ of the total nodes. LWCC and LSCC were formed mainly because of mixing services, gambling services, and crypto exchanges. The LSCC formed in past years (see Table 16) confirms this. Reuse of addresses for transferring BTCs led to the compromise of anonymity 
of bitcoin users. Thus, another feature to discriminate entities is suggested - Ratio of Transaction count and address count $(R)$. This feature gives the average number of times an address of the wallet was reused for a transaction.

Table 16: Categories and address forming LSCC

\begin{tabular}{|l|l|l|l|}
\hline Year & Address & Category & Entity name \\
\hline 2010 & 1Bw1hpkUrTKRmrwJBGdZTenoFeX63zrq33 & Unclassified & 0091107f8aaff711 \\
\hline 2011 & 1VayNert3x1KzbpzMGt2qdqrAThiRovi8 & Miner & DeepBit.net \\
\hline 2012 & 1VayNert3x1KzbpzMGt2qdqrAThiRovi8 & Miner & DeepBit.net \\
\hline 2013 & 1VayNert3x1KzbpzMGt2qdqrAThiRovi8 & Miner & DeepBit.net \\
\hline 2013 & 1P49eoo8YgWrdYmMJwo7KYAvyhJYtDfWBg & Mixer & BitcoinFog \\
\hline 2014 & 1VayNert3x1KzbpzMGt2qdqrAThiRovi8 & Miner & DeepBit.net \\
\hline 2014 & 1P49eoo8YgWrdYmMJwo7KYAvyhJYtDfWBg & Mixer & BitcoinFog \\
\hline 2015 & 1VayNert3x1KzbpzMGt2qdqrAThiRovi8 & Miner & DeepBit.net \\
\hline 2015 & 1P49eoo8YgWrdYmMJwo7KYAvyhJYtDfWBg & mixer & BitcoinFog \\
\hline 2016 & 1NxaBCFQwejSZbQfWcYNwgqML5wWoE3rK4 & Gambling & LuckyB.it \\
\hline 2016 & 1changeGhAXKoTEkMntbAe1VHh52jFQhh & Gambling & BitZillions.com \\
\hline 2016 & 19DhUuwoywejreRPhW9XWXKZTmSRNwud8x & Mixer & HelixMixer-old3 \\
\hline 2016 & 184S3jPkbwS7UJbCUYgL7VKeye5aqSKinF & Darkmarket & AlphaBayMarket \\
\hline 2019 & 1HckjUpRGcrrRAtFaaCAUaGjsPx9oYmLaZ & Exchange & Huobi.com-2 \\
\hline
\end{tabular}

\section{5. k-core decomposition}

Table 17 and 18 give the core decomposition of bitcoin users graph. The $\mathrm{k}$-core of a graph is the maximal subgraph in which every vertex has at least degree $\mathrm{k}$. The core decomposition is a set of all k-cores of a graph. Core decompositions are used to study the resilience or robustness of a network [42]. Due to the existence of single entities that captured the majority of all incoming connections, the k-cores had single nodes from 2011-2019. These single nodes were DeepBit.net (2011), SantoshiDice.com (2012-2015), Unknown wallets (2016,2018), Bittrex.com (2017), and Huobi.com-2 (2019).

Table 17: Core decomposition (2009-2015)

\begin{tabular}{|l|l|l|l|l|l|l|l|}
\hline & $\mathbf{2 0 0 9}$ & $\mathbf{2 0 1 0}$ & $\mathbf{2 0 1 1}$ & $\mathbf{2 0 1 2}$ & $\mathbf{2 0 1 3}$ & $\mathbf{2 0 1 4}$ & $\mathbf{2 0 1 5}$ \\
\hline Cores in LSCC & 5 & 9930 & 120262 & 1065542 & 347630 & 333420 & 601493 \\
\hline Cores in LWCC & 24 & 10964 & 120262 & 1065542 & 347630 & 333420 & 601493 \\
\hline Cores in full graph & 24 & 10964 & 120262 & 1065542 & 347630 & 333420 & 601493 \\
\hline
\end{tabular}


Table 18: Core decomposition (2016-2020)

\begin{tabular}{|l|l|l|l|l|l|}
\hline & $\mathbf{2 0 1 6}$ & $\mathbf{2 0 1 7}$ & $\mathbf{2 0 1 8}$ & $\mathbf{2 0 1 9}$ & $\mathbf{2 0 2 0}$ \\
\hline Cores in LSCC & 146836 & 72718 & 272896 & 1154252 & 0 \\
\hline Cores in LWCC & 112356 & 72718 & 272896 & 1154252 & 704 \\
\hline Cores in full graph & 375513 & 72718 & 272896 & 1154252 & 109080 \\
\hline
\end{tabular}
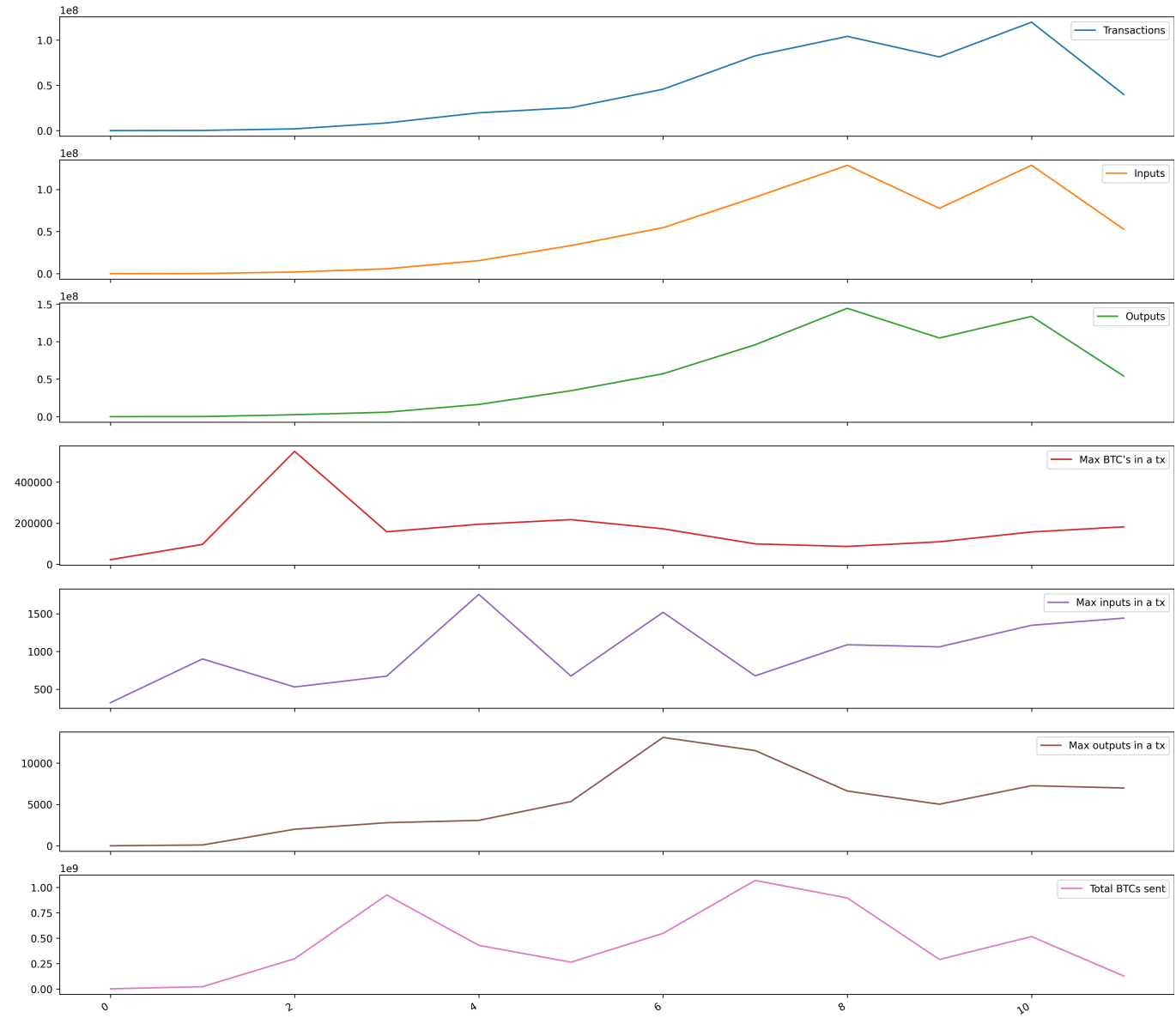

Figure 6: Distribution of transactions in Bitcoin blockchain network (2009-2020) 
- Linear regression

- Neural network: Two layers NN (units=64, activation=none)

- Convolutional neural network: Two layers (Filter $=32$, size $=1$, stride $=1$, padding $=0$ )

- LSTM: Single layer (units=32, activation=none)

The four models were trained on a single step, single output time series prediction task on the dataset of Bitcoin network characteristics from 2009-2020 viz. data mentioned in Tables 3-10 and 13-18. Results of four models on validation and test set are illustrated in Figure 7. Comparatively, dense models are better suited for the time series prediction although all four models have mean absolute error close to 0 .

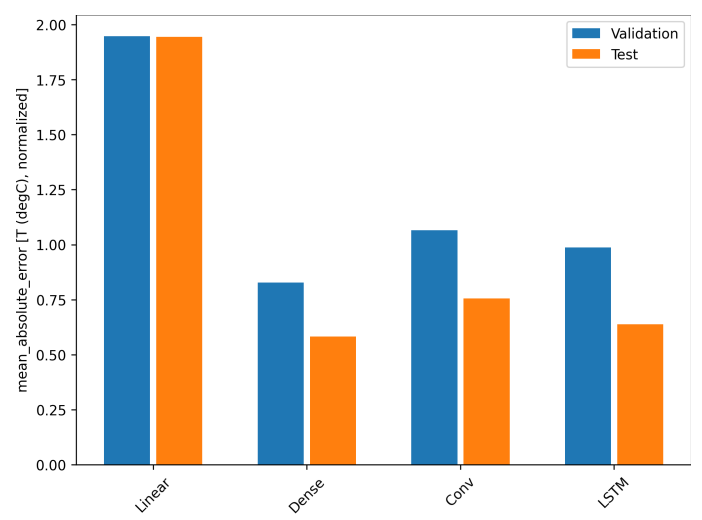

Figure 7: Performance of models on Validation and Test set

45

for

\section{act}

tr

$$
\text { a }
$$

\section{ne} 2 ne 3 in

\section{in}

Dense model was used to predict the characteristics of the Bitcoin model for the Year 2021 and results of the prediction are given in Table 19. Transactions, inputs, outputs and Max BTC's in a Tx may continue a downward trend seen in Bitcoin networks since 2019. Degree distributions could not be predicted using past data; However, centralization measures, assortativity and reciprocity were in range of previous years. Assortativity shall remain negative and reciprocity low which conforms to standard notions of Bitcoin networks. The LSCC and LWCC in Bitcoin network shall continue to domnate reaching $81 \%$ and $99 \%$ of the total network size respectively. Cores in full graphs will see a decline to 2018 levels. Overall, it can be concluded that data-driven time series analysis observes normalcy will be restored in the Bitcoin network in the year 2021 from the 2019 all time highs. 
Table 19: Prediction of Bitcoin network for Year 2021

\begin{tabular}{|l|l|l|l|l|}
\hline Year & Transactions & Inputs & Outputs & Max BTCs in a tx \\
\hline 2021 & 17916462.0 & 19343784 & 134251.34 & 15666966.0 \\
\hline Max inputs in a tx & Max outputs in a tx & Total BTCs sent & Vertex count & Edge count \\
\hline 1176.0 & 2485 & 9928711 & 269887 & 2283282 \\
\hline Edge density (S) & Edge density (D) & Power law $\backslash$ alpha in & Exp lambda in & Lognormal $\backslash$ mu in \\
\hline $4.39 \mathrm{e}-06$ & $3.64 \mathrm{e}-06$ & 0.034 & 0.13 & 0.83 \\
\hline Lognormal alpha in & Poisson in & Power law \alpha out & Exp lambda out & Lognormal $\backslash$ mu out \\
\hline 0.46 & 0.53 & 1.15 & -0.11 & 0.2 \\
\hline Lognormal alpha out & Poisson out & Diameter & Avg path length & Reciprocity \\
\hline-0.07 & -1.01 & $6.7 \mathrm{e}-02$ & $4.4 \mathrm{e}-02$ & $3.8 \mathrm{e}-02$ \\
\hline Assortativity & Centralization & Cd & Triangles (LSCC) & Nodes (LSCC) \\
\hline-0.2 & 0.99 & $4.7 \mathrm{e}-02$ & $8.6 \mathrm{e}+06$ & $1.5 \mathrm{e}+05$ \\
\hline Edges (LSCC) & AP (LSCC) & C (LSCC) & Triangles (LWCC) & Nodes (LWCC) \\
\hline $5.1 \mathrm{e}+06$ & $5.9 \mathrm{e}-03$ & $5.2 \mathrm{e}+04$ & $2.9 \mathrm{e}+07$ & $1.8 \mathrm{e}+07$ \\
\hline Edges (LWCC) & AP (LWCC) & C (LWCC) & $1.8 \mathrm{e}+07$ & Nodes (Full) \\
\hline $6.5 \mathrm{e}+07$ & $3.7 \mathrm{e}+06$ & $1.4 \mathrm{e}-04$ & Cores (Full) & $2.4 \mathrm{e}+06$ \\
\hline Edges (Full) & Cores (LSCC) & Cores (LWCC) & $4 \mathrm{e}+05$ & \\
\hline $7.3 \mathrm{e}+05$ & $2.5 \mathrm{e}+05$ & $1.65 \mathrm{e}+05$ & & \\
\hline
\end{tabular}

\subsection{Summary of Results with Discussion and lessons learnt}

- The edge density is low in both the directed graph (Edge density (D)) and the undirected graph (Edge density (S)) for the period 2009-2020 compared to social networks

- $99.8 \%$ of the total users in 2009 made at the most a single transaction this declined to $73.24 \%$ by 2020 .

- Even bitcoin users graph followed the "scale-free" property as powerlaw exponent ranged from 1.54-2.4 for in-degree distribution and from 1.42-2.7 for out-degree distribution

- LWCC and LSCC were formed mainly because of mixing services, gambling services, and crypto exchanges.

- k-cores had single nodes from 2011-2019

Comparing complex networks with bitcoins users graph, it is seen that it shares certain features with the Ethereum network. Unlike social networks (Twitter, Facebook, Actors, Directors, Co-authorship, citation), it has no giant LSCC but follows properties of "scale-free" networks. 
Table 20: Comparison with other complex networks

\begin{tabular}{|l|l|l|l|l|l|l|l|}
\hline Complex network & Hubs? & Assortativity & Small diameter? & C & $\begin{array}{l}\text { Degree } \\
\text { distribution }\end{array}$ & Giant LSCC & Edge density \\
\hline Bitcoin & Yes & $(-)$ & Yes & Low & Power law & No & Low \\
\hline Citation & NA & $(-)$ & NA & Low & Power law & NA & Low \\
\hline WWW & Yes & $(+)$ & Yes & Low & Power law & Yes & Low \\
\hline Social networking & Yes & $(-)$ & Yes & High & Power law & Yes & High \\
\hline Protein-Protein & NA & $(+)$ & NA & Low & Power law & NA & Low \\
\hline Co-authorship & NA & $(+)$ & NA & Low & No power law & NA & Low \\
\hline Ethereum & Yes & NA & Yes & Low & Power law & Yes & Low \\
\hline Film actors & NA & NA & NA & NA & Power law & NA & Low \\
\hline Company directors & NA & NA & NA & NA & No power law & NA & Low \\
\hline
\end{tabular}

With the use of deanonymizing and network analysis, common types of services on Bitcoin network datasets were able to be identified. These are listed as follows:

- Exchanges: Allow trading of BTC to fiat currencies

- Pools: Individual users combine their processing power for mining blocks

- Gambling: Allow placing of bets using BTCs

- Wallets: Store BTC private keys and balance

- Payment gateways: Allow accepting payment for services in BTCs

- Miner: Organizations competing to mine blocks

- Darknet markets: Selling and buying goods using BTCs

- Mixers: Remove traceability of BTCs from source

- Trading sites: Purchase equities using BTCs

- P2Plenders: Crowdsourcing BTCs for loans

- Faucets: Reward in BTCs to subscribers

- Explorer: Educational websites provide API to explore Bitcoin

- P2PMarket: Marketplace for second-hand goods where buyers can contact sellers, payments in BTCs

- Bond markets: Buying bonds or debt instruments in BTC 
- Affiliate marketers: Pay per click in BTC

- Video sharing: Payment in BTCs for viewing videos

- Money launderers: Convert fiat currencies to BTC

- Cyber-security providers: Provide cybersecurity products for BTC

- Cyber-criminals: Blacklisted by governments

- Ponzi: High yield investment scams

To build a system for detection of these entities in Bitcoin network and aid forensic tools, network analysis conducted in the current paper identified discriminating features. Feature list is given in Table 21. These features can be used to build a classifier for detecting or identifying illegal activities or users in Bitcoin.

Table 21: List of Features

\begin{tabular}{|l|l|}
\hline Feature symbol & Feature description \\
\hline$T_{x}$ & Total transactions in which wallet has participated \\
\hline$B$ & Current BTC present in the wallet \\
\hline$T_{x}{ }^{\text {in }}$ & Total incoming transactions to the wallet \\
\hline$T_{x}{ }^{\text {uut }}$ & Total outgoing transactions from the wallet \\
\hline$L$ & Total active life of the wallet \\
\hline$A_{w}$ & Total addresses of the wallet \\
\hline$A_{v}$ & $\begin{array}{l}\text { Average number of incoming transactions received } \\
\text { by an address of a wallet }\end{array}$ \\
\hline$T$ & $\begin{array}{l}\text { Total number of addresses sending BTC to the wallet } \\
\text { Ratio of Transaction count and address count. Gives the average number of times }\end{array}$ \\
\hline$R$ &
\end{tabular}

\section{Conclusion and Future works}

Since its launch in 2009, Bitcoin has seen a steady increase in its user base and transactions, both volume and value. As it aims to promote the exchange of value without reliance on a trusted third party, it could be speculated that the network form of the Bitcoin system should be decentralized and disconnected without any giant connected component. This would mean a robust structure. However, in reality, there are connected components in the bitcoin users graph. These components have emerged due to gambling websites, mixing services, crypto trading exchanges, and mining pools. These 
services have been easier to identify due to the high incoming and outgoing connections they have with other bitcoin users. From 2011, these entities have created giant connected components in bitcoin users graph. A result of their presence was a reduction in diameter, average path length, and radius. Additionally, "scale-free" property, was observed in bitcoin users graph as preferential attachment occurred.

The blanket of anonymity and secrecy provided by Bitcoin has made it difficult to label each and every address with a label. However, network analysis can shed light on this confidentiality and reveal the nature of the bitcoin user. There is no straightforward application of network analysis on bitcoin data as bitcoin users are identified by addresses, and a single user can have multiple addresses. This issue of multiple identities is not seen in other networks. Heuristic clustering, such as combining multi-inputs to a single transaction as a single entity, can reduce this issue to some extent and hence is commonly used in bitcoin network studies.

Even with clustering and network analysis without labeled datasets, limited progress can be made in tracing entities on the Bitcoin network. To overcome this drawback, features related to each entity can be extracted from the blockchain to train a supervised learning technique for identifying unknown wallets.

Bitcoin scenario has changed drastically in the last 3 months - e.g. Feb 20, 2020 - BTC@10k USD, March 12, 2020 - BTC@4k USD, April 2020 BTC@6k-9k, May 8 - BTC again @10k (reward halving will be happening on 11 May 2020). BTC is detaching itself from linearity of cryptocurrency market (i.e. Since last 3 months, BTC and ETH were going neck to neck in terms of percentage pricing variation). This detachment may be because of the following considerations: Pandemic Work From Home culture created opportunity for people to shift focus on stock markets and cryptocurrency markets. BTC is reemerged as a parking heaven (hedging / protection against inflation) - due to USD influx of 7 Trillion - COVID 19 stimulus printing of money - and other bailouts by governments across the World. India legalized crypto currencies from March 2020 first week (after a ban of about 2 years) and market started buzzing with large number of new players/small investors. Steady emergence of Internet of Trusted Things - which sees blockchain as a platform to build trust. 


\section{Acknowledgment}

This work was supported in part by the Raman Charpak Fellowship of the Indo-French Centre for the Promotion of Advanced Research Grant no: IFC/4132/RCF 2019/716. The authors thank VJTI Mumbai and IMT Atlantique, France for providing the lab resources. Any opinions, findings, and conclusions or recommendations expressed in this material are those of the authors and do not necessarily reflect the views of the sponsors.

[1] S. Park, S. Im, Y. Seol, J. Paek, Nodes in the bitcoin network: comparative measurement study and survey, IEEE Access 7 (2019) 57009-57022.

[2] Q. Feng, D. He, S. Zeadally, M. K. Khan, N. Kumar, A survey on privacy protection in blockchain system, Journal of Network and Computer Applications 126 (2019) 45 - 58.

[3] L. Wang, X. Shen, J. Li, J. Shao, Y. Yang, Cryptographic primitives in blockchains, Journal of Network and Computer Applications 127 (2019) $43-58$.

[4] M. Rahouti, K. Xiong, N. Ghani, Bitcoin concepts, threats, and machine-learning security solutions, IEEE Access 6 (2018) 67189-67205.

[5] S. Nakamoto, Bitcoin: A peer-to-peer electronic cash system, Tech. Rep., Manubot, 2019.

[6] S. Aggarwal, R. Chaudhary, G. S. Aujla, N. Kumar, K.-K. R. Choo, A. Y. Zomaya, Blockchain for smart communities: Applications, challenges and opportunities, Journal of Network and Computer Applications 144 (2019) $13-48$.

[7] A. A. Monrat, O. Schelén, K. Andersson, A survey of blockchain from the perspectives of applications, challenges, and opportunities, IEEE Access 7 (2019) 117134-117151.

[8] A. Ghosh, S. Gupta, A. Dua, N. Kumar, Security of Cryptocurrencies in blockchain technology: State-of-art, challenges and future prospects, Journal of Network and Computer Applications 163 (2020) 102635.

[9] R. Böhme, N. Christin, B. Edelman, T. Moore, Bitcoin: Economics, technology, and governance, Journal of economic Perspectives 29 (2) (2015) 213-38. 
[10] V. G. Reyes-Macedo, M. Salinas-Rosales, G. G. Garcia, A Method for Blockchain Transactions Analysis, IEEE Latin America Transactions 17 (07) (2019) 1080-1087.

[11] K. Toyoda, P. T. Mathiopoulos, T. Ohtsuki, A Novel Methodology for HYIP Operators Bitcoin Addresses Identification, IEEE Access 7 (2019) 74835-74848.

[12] I. Alqassem, I. Rahwan, D. Svetinovic, The anti-social system properties: Bitcoin network data analysis, IEEE Transactions on Systems, Man, and Cybernetics: Systems .

[13] X. T. Lee, A. Khan, S. S. Gupta, Y. H. Ong, X. Liu, Measurements, analyses, and insights on the entire ethereum blockchain network .

[14] F. Tschorsch, B. Scheuermann, Bitcoin and beyond: A technical survey on decentralized digital currencies, IEEE Communications Surveys \& Tutorials 18 (3) (2016) 2084-2123.

[15] D. D. F. Maesa, A. Marino, L. Ricci, The bow tie structure of the Bitcoin users graph, Applied Network Science 4 (1) (2019) 56.

[16] D. D. F. Maesa, A. Marino, L. Ricci, The graph structure of bitcoin, in: International Conference on Complex Networks and their Applications, Springer, 547-558, 2018.

[17] D. D. F. Maesa, A. Marino, L. Ricci, Data-driven analysis of Bitcoin properties: exploiting the users graph, International Journal of Data Science and Analytics 6 (1) (2018) 63-80.

[18] D. D. F. Maesa, A. Marino, L. Ricci, Uncovering the bitcoin blockchain: an analysis of the full users graph, in: 2016 IEEE International Conference on Data Science and Advanced Analytics (DSAA), IEEE, 537-546, 2016.

[19] A.-L. Barabási, et al., Network science, Cambridge university press, 2016.

[20] X. Fu, H. Yao, O. Postolache, Y. Yang, Message forwarding for WSNAssisted Opportunistic Network in disaster scenarios, Journal of Network and Computer Applications 137 (2019) 11-24. 
[21] X. Fu, G. Fortino, W. Li, P. Pace, Y. Yang, WSNs-assisted opportunistic network for low-latency message forwarding in sparse settings, Future Generation Computer Systems 91 (2019) 223-237.

[22] X. Fu, G. Fortino, P. Pace, G. Aloi, W. Li, Environment-fusion multipath routing protocol for wireless sensor networks, Information Fusion 53 (2020) 4-19.

[23] N. Szabo, Bit gold, URL https://unenumerated.blogspot.com/ 2005/12/bit-gold.html, 1970.

[24] Y. Li, U. Islambekov, C. Akcora, E. Smirnova, Y. R. Gel, M. Kantarcioglu, Dissecting Ethereum Blockchain Analytics: What We Learn from Topology and Geometry of Ethereum Graph, arXiv preprint arXiv:1912.10105 .

[25] H. Sun, N. Ruan, H. Liu, Ethereum Analysis via Node Clustering, in: International Conference on Network and System Security, Springer, 114-129, 2019.

[26] S. Ferretti, G. D'Angelo, On the Ethereum blockchain structure: A complex networks theory perspective, Concurrency and Computation: Practice and Experience (2019) e5493.

[27] P. Nerurkar, M. Chandane, S. Bhirud, Empirical analysis of synthetic and real networks, International Journal of Information Technology (2019) 1-13.

[28] P. Nerurkar, M. Chandane, S. Bhirud, Understanding structure and behavior of systems: a network perspective, International Journal of Information Technology (2019) 1-15.

[29] J. Leskovec, A. Krevl, SNAP Datasets: Stanford Large Network Dataset Collection, http://snap.stanford.edu/data, 2014.

[30] M. E. Newman, The structure and function of complex networks, SIAM review 45 (2) (2003) 167-256.

[31] M. Golosovsky, Preferential attachment mechanism of complex network growth:" rich-gets-richer" or" fit-gets-richer"?, arXiv preprint arXiv:1802.09786 . 
[32] J. MO, Social and economic networks, Princeton university press, 2010.

[33] S. Fortunato, D. Hric, Community detection in networks: A user guide, Physics Reports 659 (2016) 1-44.

[34] L. A. N. Amaral, A. Scala, M. Barthelemy, H. E. Stanley, Classes of small-world networks, Proceedings of the national academy of sciences 97 (21) (2000) 11149-11152.

[35] D. Ding, M. Conti, R. Figueiredo, Impact of country-scale Internet disconnection on structured and social P2P overlays, in: 2015 IEEE 16th International Symposium on A World of Wireless, Mobile and Multimedia Networks (WoWMoM), IEEE, 1-9, 2015.

[36] D. Ding, M. Conti, R. Figueiredo, Wide-scale internet disconnection: impact and recovery on social-based P2P overlays, IEEE Transactions on Network Science and Engineering 6 (4) (2018) 734-747.

[37] A.-L. Barabási, Network science, Philosophical Transactions of the Royal Society A: Mathematical, Physical and Engineering Sciences 371 (1987) (2013) 20120375.

[38] A. Janda, WalletExplorer. com: Smart Bicoin Block Explorer, 2016.

[39] G. Csardi, T. Nepusz, et al., The igraph software package for complex network research, InterJournal, complex systems 1695 (5) (2006) 1-9.

[40] C. S. Gillespie, Fitting heavy tailed distributions: the poweRlaw package, arXiv preprint arXiv:1407.3492 .

[41] M. O. Jackson, Social and economic networks, Princeton university press, 2010.

[42] F. D. Malliaros, C. Giatsidis, A. N. Papadopoulos, M. Vazirgiannis, The core decomposition of networks: theory, algorithms and applications, The VLDB Journal 29 (1) (2020) 61-92. 